\title{
Radiologic diagnosis of tracheoesophageal fistula in children
}

\author{
Ercan Ayaz, Mithat Haliloglu \\ Division of Pediatric Radiology, Department of Radiology, Hacettepe University School of Medicine, Ankara, Turkey \\ Contributions: (I) Conception and design: Both authors; (II) Administrative support: E Ayaz; (III) Provision of study materials or patients: M Haliloglu; \\ (IV) Collection and assembly of data: E Ayaz; (V) Data analysis and interpretation: Both authors; (VI) Manuscript writing: Both authors; (VII) Final \\ approval of manuscript: Both authors. \\ Correspondence to: Mithat Haliloglu, MD. Professor of Radiology, Department of Radiology, Hacettepe University School of Medicine, 06100 Ankara, \\ Turkey. Email: mithath@hacettepe.edu.tr.
}

\begin{abstract}
Radiological imaging plays an essential role in the diagnostic algorithm of tracheoesophageal fistula (TEF) in the prenatal and postnatal period. Although the primary imaging modality is esophagogram with water-soluble contrast, there are various imaging techniques to make the diagnosis. As ultrasound and magnetic resonance imaging (MRI) are compatible during the prenatal period, computed tomography (CT) and plain radiographs may provide additional information to contrast studies in the postpartum period. Imaging is also crucial to identify other associated anomalies and to predict the outcome. Moreover, radiological examinations are often necessary in the postoperative period to define complications and recurrent TEF. The aim of this article is two folds; first to describe indications, imaging methods and findings of congenital, acquired, and recurrent TEF and accompanying conditions to confirm the diagnosis, and second, to discuss the yield of different imaging methods in line with the literature. While describing congenital TEF, concomitant anomalies, such as esophageal atresia (EA) and tracheal agenesis were depicted systematically according to the prevalent classifications. Among acquired TEF; two specific etiologies [iatrogenic and button battery (BB) ingestion] were explained more detailed, since these entities are diagnostically challenging, required urgent management, and may have serious consequences. We also would like to emphasize pearls and challenges of the diagnostic modalities.
\end{abstract}

Keywords: Esophagogram; tracheoesophageal fistula (TEF); computed tomography (CT); ultrasonography (US); magnetic resonance imaging (MRI)

Received: 22 September 2020; Accepted: 21 October 2020; Published: 25 August 2022.

doi: $10.21037 /$ ccts-20-161

View this article at: http://dx.doi.org/10.21037/ccts-20-161

\section{Introduction}

Tracheoesophageal fistula (TEF) can be either congenital or acquired and has numerous causes. Congenital TEF is more frequently associated with esophageal atresia (EA) and very rarely with tracheal agenesis (TA). TEF and EA are relatively common congenital anomalies with a total worldwide prevalence of 2.99 per 10,000 (1). EA/TEF requires surgical repair but there are many postoperative complications after surgery, one of the most serious of which is recurrent TEF (2). Despite the developments in surgical techniques and improvement of postoperative intensive care, the incidence of recurrent TEF following EA/TEF varies from
$3 \%$ to $14 \%$ (2). Acquired TEF can be iatrogenic (e.g., due to prolonged intubation) or due to the ingestion of corrosive materials. Button battery (BB) ingestion is a major cause of acquired TEF in children. Although disk-shaped BBs have been used for almost 30 years, the major complications involving esophagus had increased almost 7 -fold in the last decade (3).

Radiological evaluation of TEF in children starts prenatally at approximately $20^{\text {th }}$ gestational week and continues in the postoperative period. Various imaging modalities have been utilized in different time courses regarding to their benefits and disadvantages. Indication and imaging modality should be individualized for each 

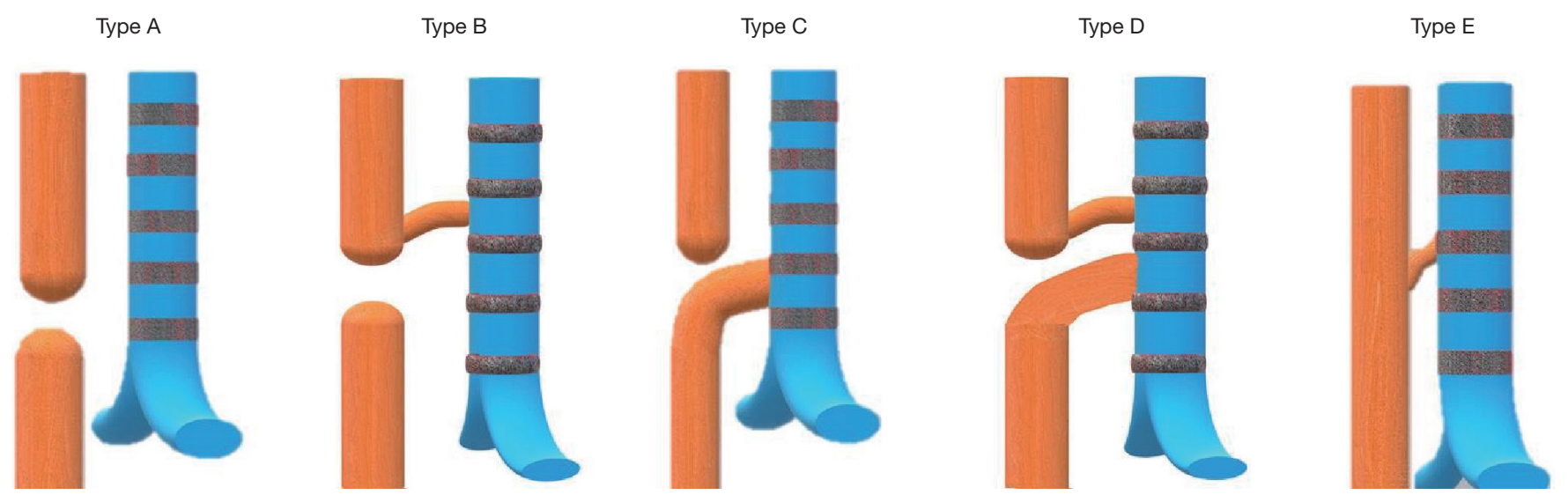

Figure 1 Illustration of Gross classification types.

patient with a consensus of radiologist, pediatric surgeon, and pediatrician. This particular review will outline the advantages and disadvantages of the radiological imaging modalities in different kinds of TEF in children. Malignant TEF, seen predominantly in adults, will not be covered in this review.

\section{Congenital TEF with or without EA}

EA is the most common congenital anomaly of the esophagus with an incidence of 1 in 2,500 to 4,500 live births (4). EA is defined as a complete interruption of the esophageal lumen (5). It can be present with or without a TEF. TEF is a congenital anomaly due to abnormal septation of the caudal foregut during the $4^{\text {th }}$ and $5^{\text {th }}$ weeks of embryonic development. The most commonly used classification system for EA/TEF is Gross classification (Figure 1) (6). According to this classification system, EA types are; Type A (isolated EA), Type B (EA with proximal TEF), Type C (EA with distal TEF), Type D (EA with proximal and distal fistulas), and Type $\mathrm{E}$ (H-type fistula). Gross Type C EA/TEF, is the most common type, comprising approximately $88.5 \%$ of cases followed by Type A (8\%), Type E (4\%), Type D (1.4\%), and Type B (0.8\%) (5).

Infants having EA with TEF are 50\% more likely than isolated EA to have congenital anomalies, with VACTERL [vertebral anomalies $(\mathrm{V})$, anal atresia $(\mathrm{A})$, cardiac anomalies (C), TEF (T), EA (E), renal anomalies (R), and limb anomalies (L)] being the most common association (7). Vertebral anomalies accompany in $60 \%$ to $90 \%$ and limb anomalies occur in $40 \%$ to $55 \%$ of cases with VACTERL association (8). In the study of Laffan et al. (9) on isolated
H-type TEF, 10\% have VACTERL association, 5\% have CHARGE association (coloboma, heart defects, choanal atresia, growth retardation and ear abnormalities) and 10\% have right-sided aortic arch. Radiographic evaluation is crucial for outlining the anomalies polydactyly, hypoplasia, length discrepancy, and to determine the need for surgical intervention. Renal anomalies affect $50 \%$ of patients; ultrasonography (US) and voiding cystourethrogram can help to detect and manage the infection and vesicoureteral reflux $(1,8)$. A wide variety of congenital lung disorders, including pulmonary and lobar agenesis, horseshoe lung and pulmonary hypoplasia, may also accompany EA/TEF (5). However, bronchial anomalies are more frequent than lung anomalies in neonates with EA/TEF (5). A bronchus that originates from the esophagus called 'esophageal bronchus' especially in H-type TEF cases is uncommon (10).

H-type TEF is a rare anomaly and comprises only $4 \%$ of the congenital TEF. H-type TEF occurs between the level of the $5^{\text {th }}$ cervical vertebra and $2^{\text {nd }}$ thoracic vertebra in up to $70 \%$ to $80 \%$ of patients and courses in an oblique route like the letter " $\mathrm{N}$ " instead of " $\mathrm{H}$ ", from the posterior wall of the trachea to the anterior wall of the esophagus $(9,11)$. The typical presentation of H-type TEF is at the early infancy period with symptoms of coughing, choking during feedings, apnea and recurrent pneumonia because of aspiration $(8,12)$. Abdominal distention due to the passage of air from the trachea to the esophagus may also occur (11). In patients with H-type TEF, chest X-ray may reveal air distended esophagus or megaesophagus as a large radiolucent column in the mediastinum (11). H-type TEF can be asymptomatic until adolescence or adulthood due to lack of aspiration, because of the possibility of a valve- 

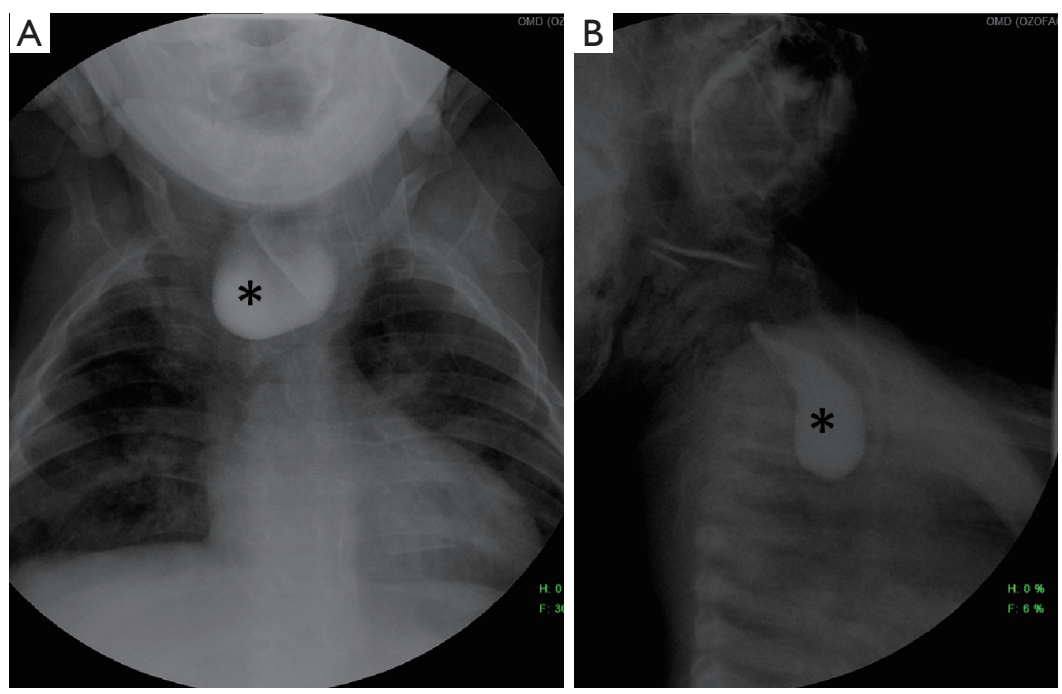

Figure 2 Posteroanterior (A) and lateral (B) pouchogram of Type A (isolated) esophagus atresia. Oral contrast fills the proximal atretic esophageal pouch (asterisk). On lateral image, a few amount of contrast is seen in the trachea anteriorly due to aspiration.

like mucosal flap which may close while swallowing (12). Delay in the diagnosis and treatment of the H-TEF may lead to significant morbidity with recurrent chest infections, bronchiectasis and failure to thrive (9).

Congenital esophageal stenosis in the distal esophagus is associated with EA/TEF. An abnormal communication between the respiratory and digestive tracts during embryonic development results in TEF and abnormal incorporation of the respiratory tissue in the esophageal wall results in congenital esophageal stenosis. It is most common in H-type TEF (21\%) (13). The incidence of congenital esophageal stenosis is varying between the range of $0.4 \%$ to $14 \%$ probably due to misdiagnosis $(14,15)$. Newman et al. (16) reported that esophageal stenosis was missed on one or more contrast swallowed fluoroscopic studies in 14 of 18 children with congenital esophageal stenosis, although retrospective evaluation revealed esophageal narrowing on the prior fluoroscopic images in the majority of cases (16). Therefore, radiologic evaluation is very important for early and accurate diagnosis, especially in fluoroscopic examination. If smooth and segmental tapering of the esophagus with proximal dilatation is seen between EA/TEF lesion and gastroesophageal junction, the presence of congenital esophageal stenosis should be considered (13). Meticulous evaluation of the esophageal movement under fluoroscopy can help to determine the actual degree and length of esophageal stenosis. Patients with achalasia like findings should be evaluated with esophagogram after EA/TEF repair to avoid delayed diagnosis of distal stenosis $(11,13)$. Newman et al. (16) suggested using a nasogastric tube to distend the esophagus during contrast esophagogram if this is not achieved with normal swallowing to improve diagnostic accuracy.

\section{Imaging methods}

Plain radiographs are generally initial imaging modality performed in these patients not only for detecting the tracheoesophageal anomalies but also associated bone anomalies. The presence of air in the esophagus on plain posteroanterior and lateral projections of the chest $\mathrm{X}$-ray is an important indicator for EA (17). If EA is suspected in the first few hours of life, placement of a flexible feeding catheter should be attempted. Contrast injection into the pouch (pouchogram) is not typically necessary for confirmation of the diagnosis and can lead to aspiration, although air may improve its identification and elucidate proximal pouch length (10) (Figure 2). A completely gasless abdomen indicates Type A or Type B EA/TEF. Gas in the stomach and bowel segments is seen in Types C, D and E.

The most useful diagnostic imaging method for TEF includes esophagogram with water-soluble contrast (12). The examination to demonstrate H-type TEF should begin with a single contrast esophagogram using pulsed digital fluoroscopy in the right lateral to slightly right anterior oblique position. Achieving full distention of the 

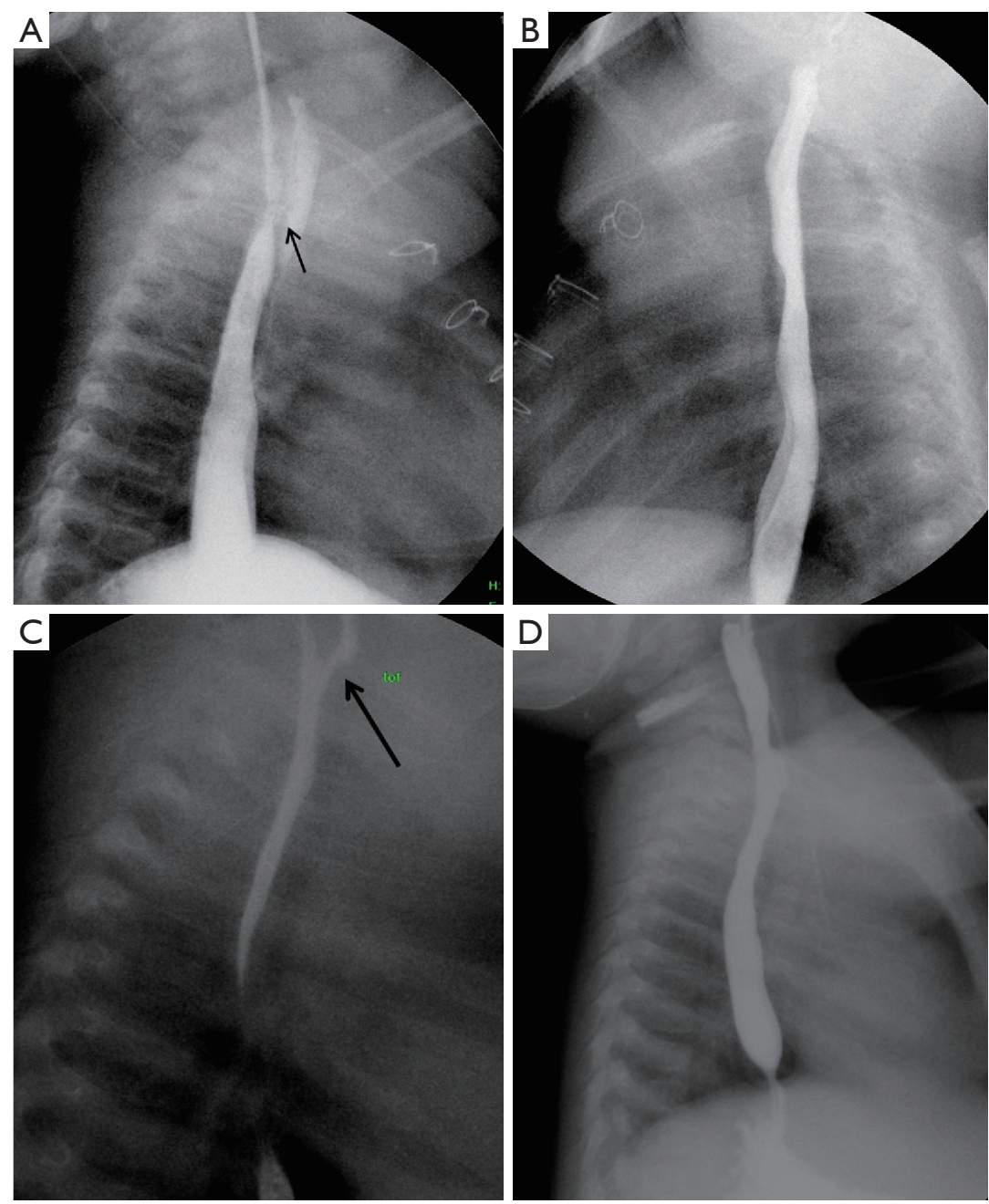

Figure 3 Fluoroscopy images of an eight-month-old boy with a history of operated tetralogy of Fallot demonstrate contrast injection into esophagus from catheter demonstrates H-type fistula (arrow) (A). On postoperative image, connection is not seen (B). Fluoroscopy images (C,D) of another seven-month-old boy with recurrent pulmonary infections after contrast injection into esophagus through catheter reveals H-type fistula (arrow) (C). Fistula is not seen in the postoperative image (D).

esophagus is the key point of the examination (10). Some authors suggested that contrast swallow can be used for the diagnosis of TEF and demonstrate fistula in up to $80 \%$ of cases, while other reports indicated that, contrast swallow studies is not an effective technique to reveal the fistula $(9,17)$. If there is suspected fistula that is not seen with contrast swallow study, the nasogastric catheter should be placed and fluoroscopic visualization of injected contrast medium at various levels of esophagus, starting from just below the carina, with the patient in lateral or proneoblique position $(18,19)$.

Slow retraction of catheter, delicate infusion of contrast and attentive fluoroscopic monitoring allow constant visualization and help to prevent excessive flow into the trachea lumen (Figure 3). It should also be stressed for the intubated patients that the endotracheal tube may obstruct the orifice of the fistula, preventing contrast material leakage from the esophagus through the fistula into the trachea. In that situation; turning the patient to the contralateral decubitus position can solve the problem (9).

There are some other disorders that can be misinterpreted as TEF on a contrast esophagogram. Laryngeal cleft, also known as laryngotracheoesophageal cleft, is a rare congenital anomaly with an estimated incidence of 1 in 

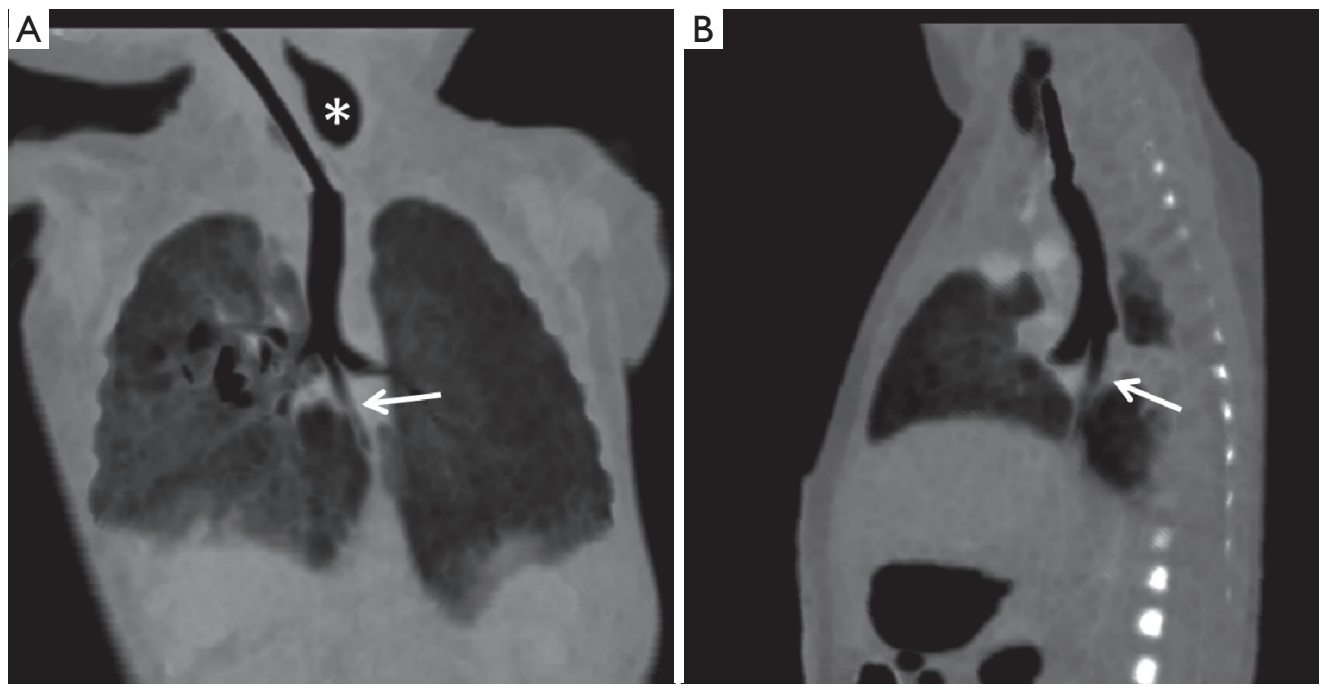

Figure 4 Coronal reformatted MinIP (A), and sagittal reformatted MinIP (B) images of Gross Type C (EA with distal TEF) disorder. Atretic proximal esophagus is seen as an air filled pouch in the cervical region (asterisk) and distal esophagus connected to the tracheobronchial system at the level of carina (arrows). MinIP, minimum intensity projection; EA, esophageal atresia; TEF, tracheoesophageal fistula.

10,000-20,000 live births (20). Laryngeal clefts should not be diagnosed as TEFs because each entity has a different management algorithm and misdiagnosis may lead to delayed definitive treatment. Although most of the laryngeal clefts are visible on laryngoscopy, in rare occasions esophagogram is required for clefts extending to the trachea (20). The main distinguishing feature between TEF and laryngeal cleft is the location of the connection. TEF has an abnormal connection between the trachea and esophagus whereas in laryngeal cleft, the fistula is between the larynx and esophagus. Involvement of the trachea can be seen in the more severe forms.

Aspiration of contrast material is another entity that can be suspected of fistulous connection when contrast material is noted in the trachea. Aspiration is observed more common in contrast swallow studies than catheter placed studies (9). Therefore, a pullback catheter esophagogram is indicated in patients who are intubated, who have a history of recurrent aspiration pneumonia and if there are associated abnormalities that significantly increase the risk of aspiration (9).

If radiological methods are unable to demonstrate fistula, bronchoscopy with simultaneous instillation of methylene blue into the esophagus can be performed as a complementary study $(17,19)$. Various anatomical features are important to define in imaging for prognosis, such as; the inter-pouch distance, position of fistula, laterality of the heart and right-sided aortic arch (21). About $2.5 \%$ of patients will have a right-sided aortic arch that should be identified before esophageal repair which is performed on the opposite side of the arch (10).

Temporal and contrast resolution of computed tomography (CT) has substantially developed over the years, and many authors have advocated the use of CT scan in preoperative evaluation of EA/TEF patients, as it is a quick non-invasive procedure that precisely delineates the anatomy of the TEF and the inter-pouch gap (22). With "minimum intensity projection (MinIP)", "multiplanar reformation (MPR)" and "virtual bronchoscopy" image reconstruction techniques, two esophageal pouches can be easily delineated on a single image (Figure 4) (23). To display TEF more clearly, examination should start with the aspiration of mucus from upper pouch and filling it with air (22). However, cost-effectiveness and associated radiation hazards raise concern regarding to utilization of this technique. Furthermore, some authors consider that CT can provide good anatomical delineation, but may not help in surgical decision making. Mahalik et al. (23) reported that CT failed to demonstrate the fistula in $20 \%$ of Gross type C cases. Consequently, preoperative CT as a diagnostic method has been a controversial topic (21-23).

Fetal ultrasound after $20^{\text {th }}$ gestational weeks can detect TEF and EA. "Pouch sign", which is caused by dilatation of the blind-ending upper esophageal segment during 
fetal swallowing, is considered to be the most consistent indicator of EA on prenatal imaging with a reported positive predictive value as high as $100 \%$ (24). However, detection of the pouch sign on ultrasound can be very challenging, as it depends on fetal position, mobility and active fetal swallowing during the examination. Additional useful findings are polyhydramnios in the setting of small or absent stomach; however, these features are nonspecific and occasionally transient in nature (1). Bradshaw et al. (25) stated that the overall sensitivity of prenatal ultrasound to identify TEF/EA was $26 \%$ with $99 \%$ specificity and $35 \%$ positive predictive value with no significant postnatal outcome. The accuracy and sensitivity of prenatal ultrasound scans increase to $57 \%$ when completed in tertiary centers. In a multicenter study of Oztan et al. (4), among 331 cases with EA with TEF, antenatal ultrasound reveals polyhydramnios in $39 \%$. On the other hand, polyhydramnios was seen in $60 \%$ of 58 patients with isolated EA. They also revealed a pouch sign in $14.9 \%$ by perinatal ultrasound (4).

The use of fetal magnetic resonance imaging (MRI) to diagnose EA is promising. Fetal MRI may improve diagnostic accuracy by the identification of pouch sign better than ultrasound however; data on both comprehensive ultrasound and MRI are limited and deserve further investigation (24). T2-weighted single shot and balanced steady-state free precession sequences are the most valuable sequences to demonstrate a fluid-filled upper esophagus. Serial sagittal images at a single level in the midline can be obtained more than 20-second intervals to evaluate fetal swallowing and the transient distension of the proximal esophageal lumen (24). Langer et al. (26) found $100 \%$ sensitivity, $80 \%$ specificity, and $83 \%$ positive predictive value using MRI to diagnose 5 cases of confirmed EA based on the non-visualization of the thoracic esophagus. Ethun et al. (24) reported that esophageal pouch sign was seen with fetal MRI in $83 \%$ of our patients with confirmed EA. They also found $100 \%$ specificity and positive predictive value using MRI to diagnose EA based on the identification of an esophageal pouch (24).

Postoperative imaging is crucial for patients. Abnormal esophageal peristalsis can be seen in $75 \%$ to $100 \%$ of children after EA surgery (100\% of those with colonic interposition) confirmed by manometry. Discoordinated contractions and segmental immotility can be assessed by contrast swallowed esophagogram (5). Occasional dysphagia after EA surgery becomes permanent in $53 \%$ to $92 \%$ of adults and daily dysphagia is reported $13 \%$ to $20 \%$. These symptoms and postoperative esophagogram findings of dysmotility are less frequent for H-type TEF. Chetcuti et al. (27) reported that $65 \%$ of patients operated for H-type TEF were admitted for GI symptoms during the first 10 years of life, but symptoms have remained in only $3 \%$ of those older than 18 years of age.

Anastomotic leak is one of the most common early complications of surgical repair, occurring in up to $17 \%$ of patients (28). Although $95 \%$ resolve spontaneously or with pleural drainage, esophageal stricture may follow in half of the cases (29). Gastroesophageal reflux is another extremely common disorder with congenital EA/TEF occurring in up to $35 \%$ to $58 \%$ of cases (17). The definitive surgical treatment is a Nissen fundoplication and $13 \%$ to $25 \%$ of patients with a history of EA/TEF require fundoplication (17). Upper GI contrast-enhanced studies can demonstrate reflux from stomach to esophagus during postoperative esophagogram.

\section{TEF after EA repair}

The communication between the esophagus and airway (trachea or bronchi) following EA repair can recur in 3\% to $14 \%$ of cases (12). Recurrent TEF occurs most frequently 2 to 18 months after initial surgery and patients generally present with cough, choking, cyanosis during feeding, and recurrent pneumonia $(18,26)$. These symptoms are often difficult to distinguish from those of tracheomalacia and gastroesophageal reflux that occur frequently in infants who already had congenital EA (18). Since the first report of Ladd about recurrent TEF in 1944 (30), there are many studies in the literature regarding to this issue.

According to Smithers et al. (2), postoperative TEFs can be divided into three types. The first type is congenital TEF that persists after repair because it was either missed or the repair attempt was insufficient. These fistulas are present immediately after the repair attempt. Most of the missed TEFs are seen in proximal $\mathrm{H}$ type fistula (2). The second type is the recurrent TEF which present in the same location as the primary congenital TEF. Majority of these fistulas are seen in Gross Type C EA (EA with distal TEF) repair with the TEF forming between the esophageal anastomosis and the tracheal diverticulum, but can also occur after proximal H-type TEF repair. The third type is the acquired TEF which occurs due to inflammation and mediastinitis in a new location on either the airway side or the digestive side (31). These fistulas include communications between the esophageal anastomosis and 

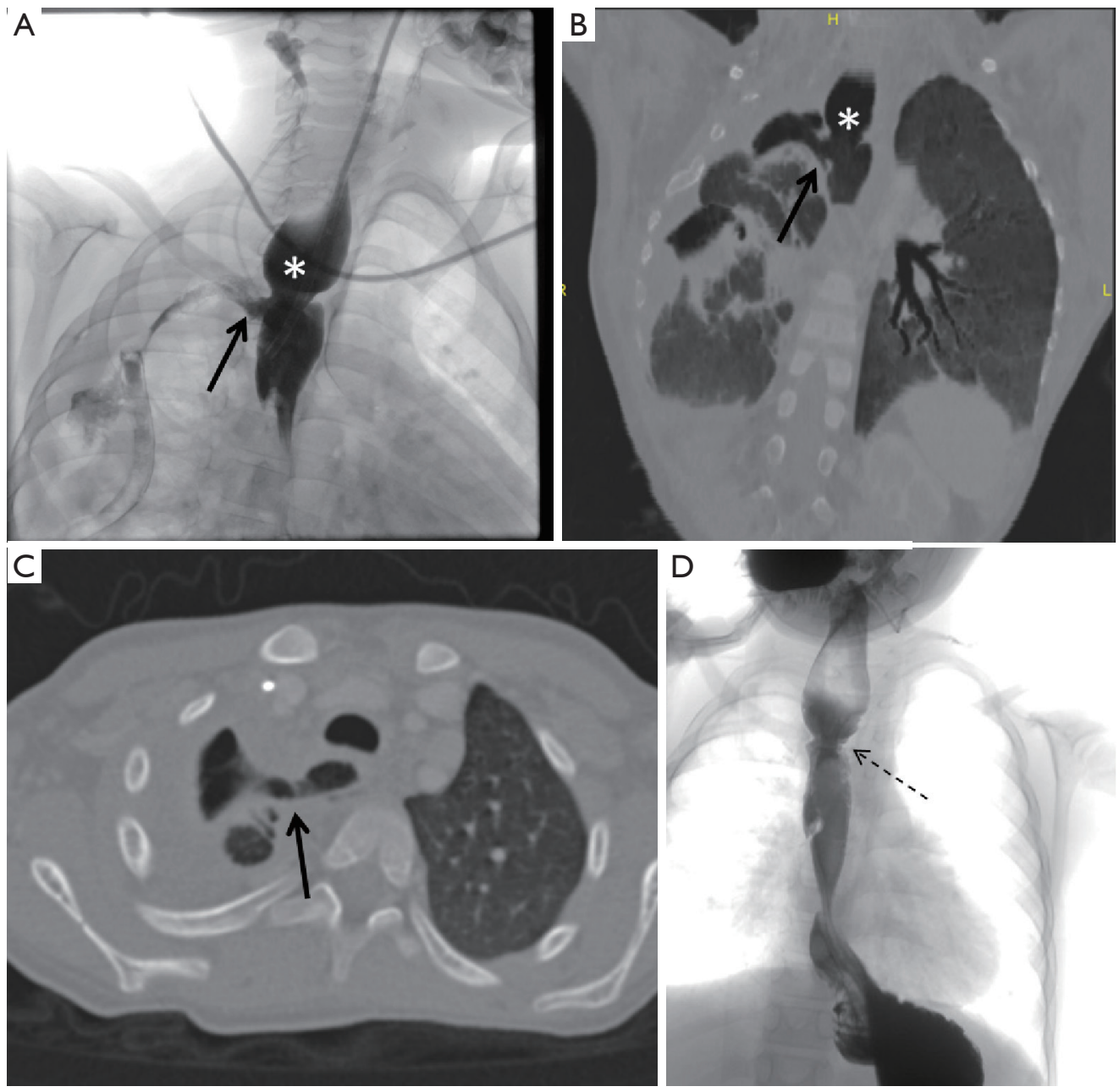

Figure 5 Fluoroscopy image (A) of a six-year-old girl with a history of EA/TEF operation and multiple endoscopic esophageal dilatations demonstrates contrast flow (arrow) from esophagus (asterisk) to right pleural cavity and drains to the pleural tube. Coronal reformatted MinIP (B) and axial CT images (C) in the same week reveal fistula (arrow) between esophagus (asterisk) and pleural cavity, pleural thickening, effusion and atelectasis of right lung. Eight months later (D), fistula was resolved without any surgery but esophageal stricture (dashed arrow) was noted. EA, esophageal atresia; TEF, tracheoesophageal fistula.

the trachea, a segmental bronchus, pleural cavity or directly pulmonary parenchyma (Figure 5).

Anastomose leak after EA repair is thought to be the leading cause of recurrent TEF (32). Recurrent TEF following anastomose leak due to initial EA repair was reported to be $45.8 \%$ to $68 \%$ in different studies $(18,32,33)$. It is also more often seen in children with concomitant congenital esophageal stenosis (14). Recurrent TEF following repair of EA is often part of a more complex complication such as; tracheomalacia that impairs airway clearance, tracheal diverticula that promote depositing of secretions and infection, esophageal strictures that increase the pressure gradient between the esophagus and the trachea, and gastroesophageal reflux that causes airway irritation (5). Smithers et al. (2) suggest that using the terms of "esophagobronchial fistula" and "esophagopulmonic fistula" to define postoperative fistulas may be more accurate for those complex cases. Additionally, stricture and severe inflammation around the anastomosis are common findings and can be demonstrated on esophagogram after EA repair (31). For postoperative strictures, balloon dilation is a safe procedure in the treatment of strictures, though it is considered a risk factor for recurrent TEF development (31). 
The primary and the most efficient imaging method for recurrent TEF is esophagogram with water-soluble contrast material given through the nasogastric catheter. Alternative imaging method is bronchoscopy; however, it is reserved for inconclusive cases due to invasive procedure. In the study of Wang et al. (18), the sensitivity of esophagogram and bronchoscopy regarding to recurrent TEF is $91.4 \%$ and $88.6 \%$ respectively. Bronchoscopic diagnosis and catheter placement to the recurrent TEF is more challenging than primary TEF because of the abnormal localization of the fistulas especially for acquired TEF and missed congenital TEF (31). If these methods are ineffective to demonstrate fistula, then diluted methylene blue is injected into the old pit of the initial TEF division with a gastroscope placed in the esophagus (19).

Recurrent TEF is difficult to diagnose and may be missed quite easily on routine contrast swallowed upper GI series, especially if a prone pull-back method is not used (18). The best way to demonstrate fistula clearly is to place a catheter down the esophagus to the gastroesophageal junction, place the infant prone, and inject contrast medium into the esophagus while the tube is pulled back into the proximal esophagus $(18,19)$.

In patients with a second recurrence of TEF, both re-anastomosis and esophageal replacement (colonic interposition) had been successfully applied in previous studies, but there are controversies in the literature regarding to preservation of native esophagus (18).

\section{Tracheal agenesis with TEF}

Tracheal agenesis is a rare and essentially lethal congenital abnormality with approximately 200 cases reported in the literature since its initial description in 1900 (34). Commonly used classification system for tracheal agenesis was defined by Floyd et al. as follows; type I: agenesis of the proximal trachea with a normal caudal segment of the distal trachea and a TEF, type II: total TA with normal main bronchi fusing in the midline at the carina (a tracheoesophageal fistula may or may not be present), type III: complete TA with a separate origin of the main bronchi from the esophagus (35). Floyd type II is the most common type of TA and TEF accompanies tracheal agenesis in $94 \%$ of patients $(35,36)$.

Prenatal presentation of tracheal agenesis is consistent with congenital high airway obstruction syndrome (CHAOS) if a connection with esophagus is not present.
In cases with co-existing TEF; lung fluid can pass through the TEF to the stomach or amniotic sac and the lungs have a normal sonographic appearance (36). Polyhydramnios is reported prenatally in up to $50-72 \%$ of patients with tracheal agenesis, with or without esophageal distention but these findings are nonspecific and raise the possibility of an EA with TEF $(35,36)$. Esophagogram and CT can be used postnatally to depict types of tracheal agenesis with TEF, with additional findings including low carinal position, flattened carina with horizontal bronchi and broadened mainstem bronchi (Figure 6) (37). MRI, both fetal and neonatal, may be useful for describing tracheoesophageal abnormalities if the esophagus is sufficiently distended (36).

\section{latrogenic TEF due to tracheal intubation}

Prolonged tracheal intubation is one of the main causes of TEF, even though the introduction of high volume and lowpressure endotracheal tube cuffs has reduced the incidence of this complication (38). The incidence is between $0.3 \%$ and $3 \%$ in patients with prolonged mechanical ventilation and tracheostomy does not seem to be effective to reduce the risk of TEF (28). Chronic pressure on the posterior membranous wall of trachea by the hyper-inflated endotracheal tube cuff along with a rigid nasogastric tube in the esophagus produces ischemic necrosis that affects both the anterior wall of the esophagus and the posterior wall of trachea. Frequently, tracheal stenosis co-exists at the level of connection (38). Possible risk factors are high airway pressure during mechanical ventilation, excessive mobility of the endotracheal tube, prolonged time of intubation, steroid treatment, poor nutritional status, chronic hypoxia in cardiopulmonary diseases, chronic anemia, sepsis and gastroesophageal reflux $(28,39)$.

On imaging, chest radiographs-especially lateral projection-may show the dilatation of the esophagus distal to the fistula. It can also reveal the findings of pulmonary aspiration of gastroesophageal content (40). The esophagogram is especially useful where endoscopy is not available. Ingestion of a small amount of watersoluble contrast agent usually reveals the site of the fistula and the abnormal route of the contrast agent into the tracheobronchial tree (38). CT is generally not required for the diagnosis of acquired TEF due to intubation, but it can be performed for complications such as mediastinitis and pulmonary abscess or necrotizing pneumonia. 

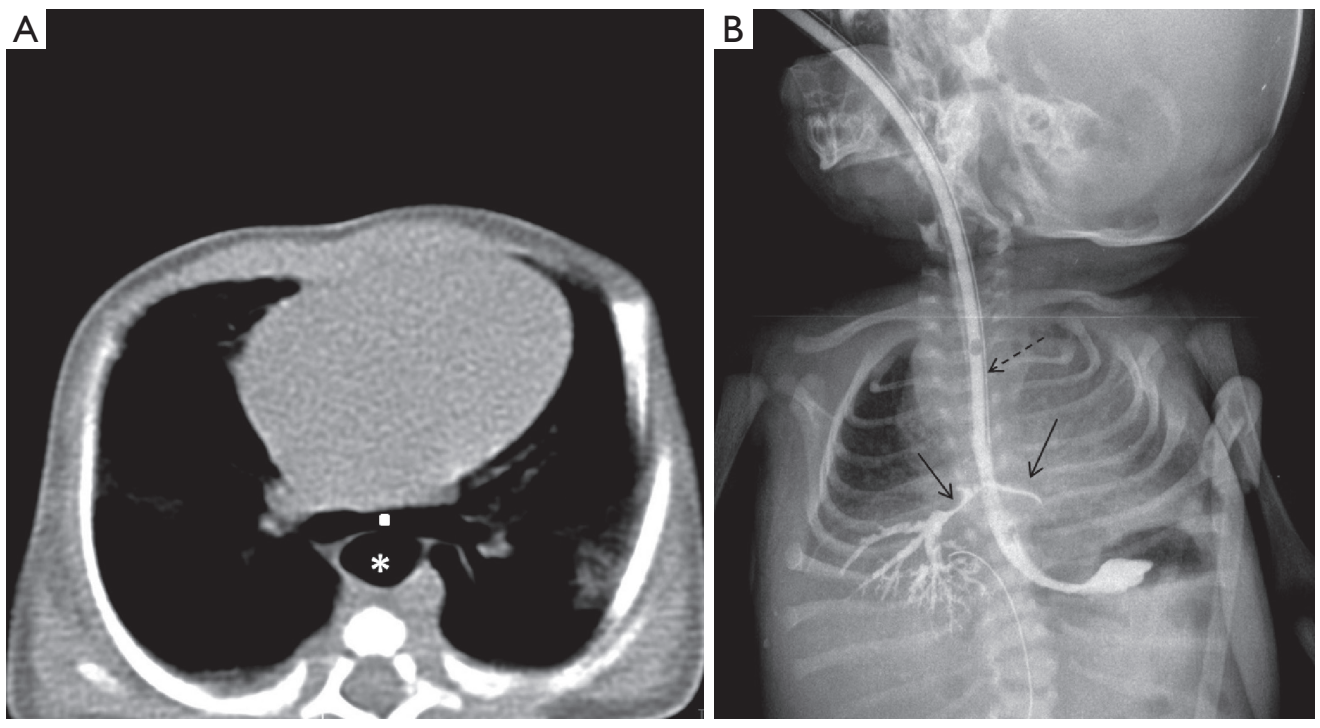

Figure 6 Newborn girl born with respiratory distress could not intubate via tracheal intubation. On chest CT (A), proximal trachea was not seen and carina (square) communicated with distal esophagus (asterisk). On fluoroscopy (B), contrast injected through nasoesophageal tube (dashed arrow) and flows into both main bronchi (arrows) and right lower bronchi. The baby died at the sixth day and the autopsy confirmed the diagnosis of Floyd type 2 tracheal agenesis.

\section{Acquired TEF due to BB ingestion}

The use of button batteries is becoming more common in electronic devices, such as children's toys, and the potential damage after ingestion has been well documented (41). The recent increase in morbidity and mortality associated with $\mathrm{BB}$ ingestions is presumably due to the increased diameter of button batteries on the market and a transition to lithium cells (42). Children less than 5 years of age who ingest batteries $20 \mathrm{~mm}$ or greater in diameter are at particular risk for catastrophic complications (43). Pugmire et al. (42) reported that the vast majority (92.6\%) of button batteries retained in esophagus are $20 \mathrm{~mm}$ or larger in diameter, in contrast to batteries that pass distal to the esophagus being measured $13.7 \pm 1.6 \mathrm{~mm}$ on average diameter. The most common site of esophageal impaction is the distal esophagus (40.7\%). Of children who ingested batteries $20 \mathrm{~mm}$ or larger in diameter, only $1.8 \%$ had major complications including TEF and perforation (42).

Radiography remains the primary imaging modality to assess suspected BB ingestions, as well as other foreign body ingestions, but additional imaging modalities should be performed for associated complications (42). Owing to urgent management of treatment course by immediate endoscopic removal within 2 hours, fluoroscopic upper GI examinations are rarely performed for diagnosis. In patients with suspected TEF, contrast studies can be used to demonstrate the connection, however, it may not be easy and dangerous because of strictures, mucosal fragility and excessive inflammation (44).

In 2015, the North American Society for Pediatric Gastroenterology, Hepatology and Nutrition (NASPGHAN) suggested a new algorithm in terms of imaging modality, CT or MRI, to manage ingested button batteries (3). In patients with any esophageal injury is identified on endoscopy following BB ingestion, the guidelines recommend considering $\mathrm{CT}$ angiography of the chest to assess for aortic injury and MRI to assess for inflammatory changes near the esophagus and aorta (3).

\section{Conclusions}

Although the prognosis of newborns with TEF with or without EA has improved over the years, with a survival rate of $85 \%$ to $95 \%$, associated anomalies and postoperative complications change long-term outcomes significantly (45). Radiological evaluation is crucial to reveal associated anomalies and complications, to predict the outcome and to prevent the delay in appropriate treatment approach, which can lead to long-term complications. The appropriate selection of optimal imaging methods for each case with 
an attentive examination would provide better patient management.

\section{Acknowledgments}

Funding: None.

\section{Footnote}

Provenance and Peer Review: This article was commissioned by the Guest Editor (Tutku Soyer) for the series "Tracheoesophageal Fistula" published in Current Challenges in Thoracic Surgery. The article has undergone external peer review.

Conflicts of Interest: Both authors have completed the ICMJE uniform disclosure form (available at https://ccts. amegroups.com/article/view/10.21037/ccts-20-161/coif). The series "Tracheoesophageal Fistula" was commissioned by the editorial office without any funding or sponsorship. The authors have no other conflicts of interest to declare.

Ethical Statement: The authors are accountable for all aspects of the work in ensuring that questions related to the accuracy or integrity of any part of the work are appropriately investigated and resolved.

Open Access Statement: This is an Open Access article distributed in accordance with the Creative Commons Attribution-NonCommercial-NoDerivs 4.0 International License (CC BY-NC-ND 4.0), which permits the noncommercial replication and distribution of the article with the strict proviso that no changes or edits are made and the original work is properly cited (including links to both the formal publication through the relevant DOI and the license). See: https://creativecommons.org/licenses/by-nc-nd/4.0/.

\section{References}

1. Lee S. Basic Knowledge of tracheoesophageal fistula and esophageal atresia. Adv Neonatal Care 2018;18:14-21.

2. Smithers CJ, Hamilton TE, Manfredi MA, et al. Categorization and repair of recurrent and acquired tracheoesophageal fistulae occurring after esophageal atresia repair. J Pediatr Surg 2017;52:424-30.

3. Kramer RE, Lerner DG, Lin T, et al. Management of ingested foreign bodies in children: a clinical report of the NASPGHAN Endoscopy Committee. J Pediatr
Gastroenterol Nutr 2015;60:562-74.

4. Oztan MO, Soyer T, Oztorun CI, et al. Outcome of very low and low birth weight infants with esophageal atresia: results of the Turkish esophageal atresia registry. Eur J Pediatr Surg 2021;31:226-35.

5. Kovesi T, Rubin S. Long-term complications of congenital esophageal atresia and/or tracheoesophageal fistula. Chest 2004;126:915-25.

6. Gross RE. The Surgery of Infancy and Childhood. WB Saunders, Philadelphia, 1953.

7. Haller JO, Berdon WE, Levin TL, et al. Tracheoesophageal fistula (H-type) in neonates with imperforate anüs and the VATER association. Pediatr Radiol 2004;34:83-5.

8. Solomon BD, Baker LA, Bear KA, et al. An approach to the identification of anomalies and etiologies in neonates with identified or suspected VACTERL (Vertebral Defects, Anal Atresia, Tracheo-Esophageal Fistula with Esophageal Atresia, Cardiac Anomalies, Renal Anomalies, and Limb Anomalies) association. J Pediatr 2014;164:451-7.e1.

9. Laffan EE, Daneman A, Ein SH, et al. Tracheoesophageal fistula without esophageal atresia: are pull-back tube esophagograms needed for diagnosis? Pediatr Radiol 2006;36:1141-7.

10. Ellis WD. Esophagus-Congenital and Neonatal Abnormalities. In: Coley BD. editor. Caffey's Pediatric Diagnostic Imaging. Elsevier, Philadelphia, 2019;901-10.

11. Boybeyi O, Köse M, Ersöz DD, et al. Achalasia-like findings in a case with delayed diagnosis of $\mathrm{H}$-type tracheoesophageal fistula. Pediatr Surg Int 2008;24:965-9.

12. Wetzler G, Jo I, Breglio K, et al. Adolescent presentation of congenital tracheoesophageal fistula. J Pediatr Gastroenterol Nutr 2017;64:e76-e79.

13. Yoo HJ, Kim WS, Cheon JE, et al. Congenital esophageal stenosis associated with esophagealatresia/ tracheoesophageal fistula: clinical and radiologic features. Pediatr Radiol. 2010;40:1353-9.

14. Kawahara H, Imura K, Yaqi H, et al. Clinical characteristics of congenital esophageal stenosis distal to associated esophageal atresia. Surgery 2001;129:29-38.

15. Ibrahim AH, Al Malki TA, Hamza AF, et al. Congenital esophageal stenosis associated with esophageal atresia: new concepts. Pediatr Surg Int 2007;23:533-7.

16. Newman B, Bender TM. Esophageal atresia/ tracheoesophageal fistula and associated congenital esophageal stenosis. Pediatr Radiol 1997;27:530-4.

17. Karnak I, Senocak ME, Hiçsönmez A, et al. The diagnosis and treatment of $\mathrm{H}$-type tracheoesophageal fistula. J 
Pediatr Surg 1997;32:1670-4.

18. Wang J, Zhang M, Pan W, et al. Management of recurrent tracheoesophageal fistula after esophageal atresia and follow-up. Dis Esophagus 2017;30:1-8.

19. Coran AG. Redo esophageal surgery: the diagnosis and management of recurrent tracheoesophageal fistula. Pediatr Surg Int 2013;29:995-9.

20. Jesse C, Jonathan S, Jeremy N, et al. A rare case of laryngeal cleft in association with VACTERL and malrotation. Radiol Case Rep 2018;14:315-9.

21. Fitoz S, Atasoy C, Yagmurlu A, et al. Three dimensional CT of congenital esophageal atresia and distal tracheoesophageal fistula in neonates: preliminary results. AJR Am J Roentgenol 2000;175:1403-7.

22. Garge S, Rao KL, Bawa M. The role of preoperative CT scan in patients with tracheoesophageal fistula: a review. J Pediatr Surg 2013;48:1966-71.

23. Mahalik SK, Sodhi KS, Narasimhan KL, et al. Role of preoperative 3D CT reconstruction for evaluation of patients with esophageal atresia and tracheoesophageal fistula. Pediatr Surg Int 2012;28:961-6.

24. Ethun CG, Fallon SC, Cassady CI, et al. Fetal MRI improves diagnostic accuracy in patients referred to a fetal center for suspected esophageal atresia. J Pediatr Surg. 2014;49:712-5.

25. Bradshaw CJ, Thakkar H, Knutzen L, et al. Accuracy of prenatal detection of tracheoesophageal fistula and oesophageal atresia. J Pediatr Surg. 2016;51:1268-72.

26. Langer JC, Hussain H, Khan A, et al. Prenatal diagnosis of esophageal atresia using sonography and magnetic resonance imaging. J Pediatr Surg. 2001;36:804-7.

27. Chetcuti P, Myers NA, Phelan PD, et al. Adults who survived repair of congenital oesophageal atresia and tracheo-oesohageal fistula. BMJ 1988;297:344-6.

28. Couraud L, Ballester MJ, Delaisement C. Acquired tracheoesophageal fistula and its management. Semin Thorac Cardiovasc Surg 1996;8:392-9.

29. Engum SA, Grosfeld JL, West KW, et al. Analysis of morbidity and mortality in 227 cases of esophageal atresia and/or tracheoesophageal fistula over two decades. Arch Surg 1995;130:502-8.

30. Ladd WE. The surgical treatment of esophageal atresia and tracheoesophageal fistulas. N Engl J Med 1944;230:625-37.

31. Boybeyi Türer Ö, Tanyel FC, et al. Acquired tracheoesophageal fistula after esophageal atresia repair. Balkan Med J 2019;37:47-9.

32. Koivusalo AI, Pakarinen MP, Lindahl HG, et al.
Revisional surgery for recurrent tracheoesophageal fistula and anastomotic complications after repair of esophageal atresia in 258 infants. J Pediatr Surg 2015;50:250-4.

33. Ghandour KE, Spitz L, Brereton RJ, et al. Recurrent tracheo-oesophageal fistula: experience with 24 patients. J Paediatr Child Health 1990;26:89-91.

34. de Groot-van der Mooren MD, Haak MC, Lakeman $\mathrm{P}$, et al. Tracheal agenesis: approach towards this severe diagnosis. Case report and review of the literature. Eur J Pediatr 2012;171:425-31.

35. Floyd J, Campbell DC Jr, Dominy DE. Agenesis of the trachea. Am Rev Respir Dis 1962;86:557-60.

36. Coleman AM, Merrow AC, Elluru RG, et al. Tracheal agenesis with tracheoesophageal fistulae: fetal MRI diagnosis with confirmation by ultrasound during an ex utero intrapartum therapy (EXIT) delivery and postdelivery MRI. Pediatr Radiol 2013;43:1385-90.

37. Strouse PJ, Newman B, Hernandez RJ, et al. CT of tracheal agenesis. Pediatr Radiol 2006;36:920-6.

38. Paraschiv M. Tracheoesophageal fistula - a complication of prolonged tracheal intubation. J Med Life 2014;7:516-21.

39. Payne DK, Anderson WM, Romero MD, et al.

Tracheoesophageal fistula formation in intubated patients. Chest 1990;98:161-4.

40. Grillo HC. Surgery of the tracheal and bronchi, Cap. XII, 2004, BC Decker Inc. Hamilton London.

41. Chessman R, Verkerk M, Hewitt R, et al. Delayed presentation of button battery ingestion: a devastating complication. BMJ Case Rep 2017;2017:bcr2017219331.

42. Pugmire BS, Lin TK, Pentiuk S, et al. Imaging button battery ingestions and insertions in children: a 15-year single-center review. Pediatr Radiol 2017;47:178-85.

43. Litovitz T, Whitaker N, Clark L, et al. Emerging battery ingestion hazard: clinical implications. Pediatrics 2010;125;1168-77.

44. Imamoğlu M, Cay A, Koşucu P, et al. Acquired tracheoesophageal fistulas caused by button battery lodged in the esophagus. Pediatr Surg Int 2004;20:292-4.

45. Al-Salem AH, Kothari M, Oquaish M, et al. Morbidity and mortality in esophageal atresia and tracheoesophageal fistula: a 20-year review. Ann Pediatr Sur 2013;9:93-8.

doi: $10.21037 /$ ccts-20-161

Cite this article as: Ayaz E, Haliloglu M. Radiologic diagnosis of tracheoesophageal fistula in children. Curr Chall Thorac Surg 2022;4:25. 